\title{
Uterine Cervical Melanocytic Tumours-A Single Institution Experience with Review of Literature
}

\author{
Indu R. Nair ${ }^{1}$, Anupama Rajanbabu' ${ }^{2}$, Annie Jojo', Shruthi Shivdas ${ }^{2}$, \\ Vijaykumar Kottarathil2, K. Radhamany ${ }^{3}$ \\ ${ }^{1}$ Department of Pathology, Amrita School of Medicine, Amrita Institute of Medical Sciences, Amrita Vishwa \\ Vidyapeetham (Amrita University), Kochi, India \\ ${ }^{2}$ Department of Gynaecological Oncology, Amrita School of Medicine, Amrita Institute of Medical Sciences, \\ Amrita Vishwa Vidyapeetham (Amrita University), Kochi, India \\ ${ }^{3}$ Department of Gynaecology, Amrita School of Medicine, Amrita Institute of Medical Sciences, Amrita Vishwa \\ Vidyapeetham (Amrita University), Kochi, India \\ Email: drinduharikrishnan@gmail.com
}

Received 20 February 2016; accepted 9 April 2016; published 12 April 2016

Copyright (C) 2016 by authors and Scientific Research Publishing Inc.

This work is licensed under the Creative Commons Attribution International License (CC BY).

http://creativecommons.org/licenses/by/4.0/

c) (i) Open Access

\begin{abstract}
Primary melanocytic tumours of the uterine cervix are rare. These include blue nevi and malignant melanoma. Blue nevi are benign melanocytic lesions which occur as incidental finding. Primary cervical melanomas are rare mucosal melanomas with aggressive behaviour. In small biopsies, they can mimic poorly differentiated carcinomas. Here we present the cases of melanocytic tumours reported from our institute during the last 6 years.
\end{abstract}

\section{Keywords}

Blue Nevus, Primary Uterine Cervical Melanoma

\section{Introduction}

Melanocytic tumours of the uterine cervix are rare [1]. These include blue nevus and malignant melanoma [2]. Blue nevi are extremely rare incidental findings. Cervical melanomas constitute less than $2 \%$ of all mucosal melanomas and less than 5\% of genital melanomas [3]. A literature review showed that from, 1889 to date only 89 cases of primary cervical malignant melanomas have been reported. The first well documented case was reported by Taylor and Tuttle in 1944 [4]. Here we present a detailed review of the melanocytic lesions reported 
so far in literature with an account of the cervical melanocytic tumours diagnosed in our surgical oncology centre during the last 6 years (Jan 2009 to Dec 2014). Among the total 3416 hysterectomies, 3 blue nevi and 1 primary cervical melanoma were reported.

\section{Case Report-Cervical Blue Nevus}

We received 3416 hysterectomy specimens in the department of pathology, Amrita Institute, Kochi, during the last 6 years, from the departments of Surgical oncology and Gynaecology. There were 3 cases of common blue nevi. All the 3 patients were in the perimenopausal age group (42 - 51 yrs) and underwent hysterectomy for fibroids. Grossly cervixes were unremarkable in 2, and showed congestion in one. Microscopically all 3 cases showed cervix with unremarkable ecto cervical epithelium. Sub epithelium showed scattered spindly/elongated cells with moderate cytoplasm and uniform round/oval nuclei. Cytoplasm showed brownish black pigment granules, which were removed with Melanin bleach. Iron stain was negative. None of the patients had co existing melanomas.

\section{Case Report-Cervical Malignant Melanoma}

A 62 year old female presented with complaints of postmenopausal bleeding of 2 wks duration .Pelvic examination revealed a large exophytic growth involving the entire cervix, extending to parametrium. Cervical punch biopsy was done, which showed fragmented tissue with ulcerated epithelium and an infiltrating neoplasm composed of cells arranged in diffuse pattern with scanty intervening stroma. No individual cell keratinisation/keratin pearls/cytoplasmic pigments were seen. Diagnosis of a non keratinizing large cell squamous cell carcinoma was offered. Subsequently, the patient received concurrent chemo radiation followed by brachytherapy. After one month of completing radiation pelvic examination revealed residual disease without parametrial extension. Radical hysterectomy was done, with bilateral pelvic lymph node dissection. Hysterectomy specimen showed an irregular grey white growth with heamorrhagic areas involving the cervix and measuring $4.7 \mathrm{~cm}$ in maximum dimension. On microscopic examination, sections from cervix showed ecto cervical epithelium with focal ulceration and an underlying neoplasm composed of similar cells as in small biopsy. There was focal junctional activity. In addition, were seen sheets of spindly/epithelioid cells with abundant brownish pigment in the cytoplasm (removed with Melanin bleach). A Masson Fontana stain was done in the previous small biopsy, which revealed the melanin pigment in many of the cells. Immuno histo chemistry showed the neoplastic cells to be positive for S100 and HMB 45 (Figure 1), and negative for cytokeratin and synaptophysin, confirming the diagnosis of malignant melanoma. Resected margins and lymph nodes were free. A detailed clinical examination was done which ruled out melanoma elsewhere in the body. Now, the patient is on regular follow up and there is no evidence of disease after 3 years of completing the treatment.

\section{Discussion}

The origin of melanocytes in the Mullerian tract is still debated. There are different theories regarding their ori-

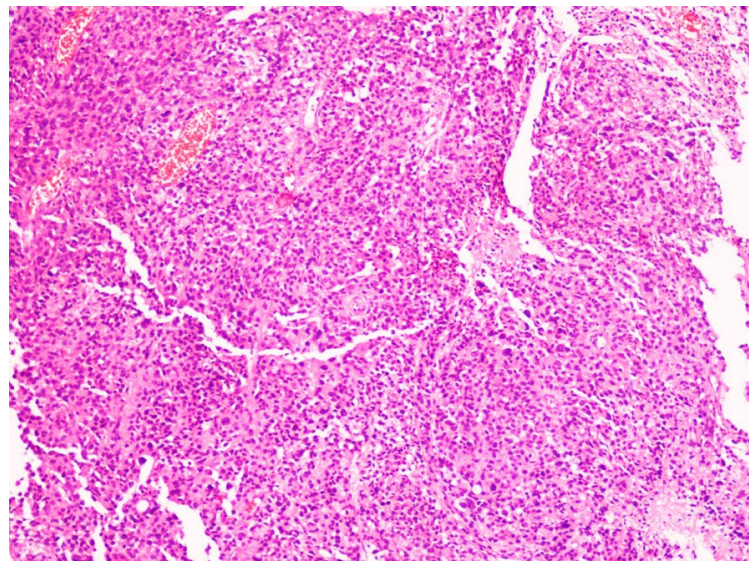

(a)

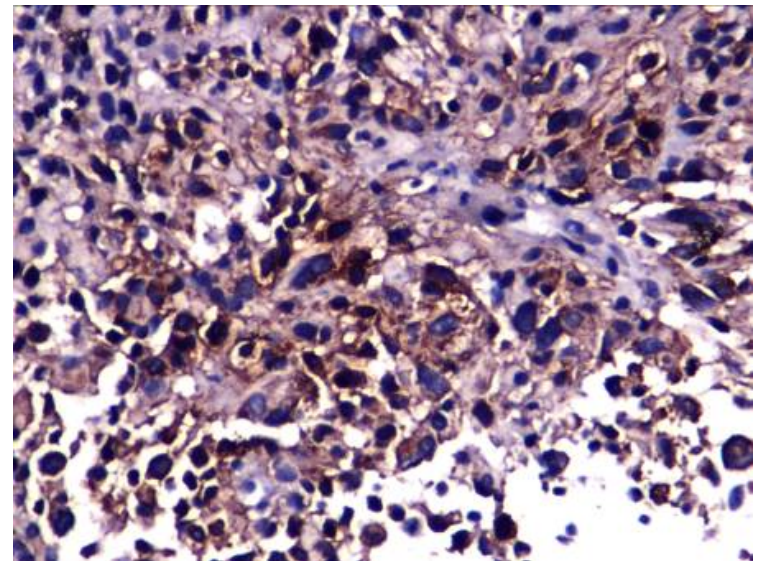

(b)

Figure 1. (a) Cervical malignant melanoma-H \& EX20; (b) IHC for HMB-45 positive in the tumour cells. 
gin. One of the theories is that melanocytic precursors migrate aberrantly during embryogenesis towards the mullerian epithelium [5]. Uehera et al. detected microscopic foci of scattered stromal melanocytes by performing serial sections in $28 \%$ of 189 benign hysterectomy specimens and termed it "stromal melanocytic foci" [6]. Another theory is that they may be melanogenically transformed Schwann cells of stromal nerves. This hypothesis has been supported by ultra structural studies showing some Schwannian features in these cells [7].

Melanocytic lesions are described in extracutaneous sites like oral and esophageal mucosa, maxillary sinus, conjunctiva, meninges, vagina etc [3]. Cervix is a very rare site for these lesions. WHO has described [2] primary melanocytic lesions in cervix-blue nevus and malignant melanoma. Blue nevus of the endocervix was first described in 1959, with only about 100 cases reported so far, in literature. Patel and Bhagavan [8] analysed 2500 hysterectomy specimens and reported only 3 cases of blue nevus in them. They are microscopic lesions less than $1 \mathrm{~cm}$ in diameter and are often missed during gross examination. If seen grossly, they can be mistaken for either endometriosis or haemangioma. Microscopically, the features are similar to the common blue nevus of the skin. A histological continuum exists from common blue nevi to cellular blue nevi. In common blue nevus, a vaguely nodular collection of poorly melanized spindled melanocytes and deeply pigmented dendritic melanocytes within thickened collagen bundles is seen. Scattered melanophages are usually noted. Cytological atypia or mitotic activity is absent. Whereas, in cellular blue nevus, a well-demarcated nodule formed by fascicles and nests of tightly packed, spindled to oval melanocytes with scattered melanophages is seen. Histochemically, the cells are positive for Masson-Fontana that demonstrates melanin pigment and the pigment is removable with melanin bleach. Immunohistochemically Melan-A, S-100 and HMB-45 are positive, which confirm the melanocytic origin of cells. Cellular blue nevi are even rarer in cervix. Literature search showed only 2 cases of cellular blue nevi in the uterus. Common blue nevi are clinically benign, and are incidental findings in hysterectomies, done for other indications. Cellular blue nevi are also clinically benign. Because of their large size, biopsy and excision tend to be performed more often on cellular blue nevi. Rare cases of malignant melanoma have been reported to arise in cellular blue nevi [9].

Primary mucosal melanomas represent only about $1.4 \%$ of all melanomas with higher rates among women compared to men (may be because of increased incidence of genital melanomas) [3]. Most of the patients are older than 60 years [4]. Several factors are implicated in the etiopathogenesis of melanomas. They include viruses like human papilloma virus [10] and toxins like formaldehyde [11]. Female genital tract melanomas account for $18 \%$ of all mucosal melanomas. Among female genital tract tumours, the most common is vulvar melanoma (76.7\%) followed by vaginal (19.8\%), while cervical melanoma is the least common [12]. Primary cervical melanomas constitute less than $2 \%$ of all mucosal melanomas and less than $5 \%$ of genital melanomas. These are very rare, with less than 100 cases reported in the literature so far .Usually cervical involvement occurs secondarily in vaginal melanomas. A detailed review of literature showed 88 reported cases of primary cervical melanomas, from 1889 to 2015 September (Table 1). This include the 78 cases reviewed by Pusceddu et al. 1 in 2012.A literature search was done to find out the number of cases reported during the period 2013 to 2015 , which revealed another 10 cases. Ours will be 89th case in this series. According to this review, cervical melanomas occur commonly in women older than 50 years. Majority of patients are symptomatic at the time of diagnosis, with vaginal bleeding being the most common symptom followed by vaginal discharge. Inspection usually shows blackish/bluish exophytic cervical mass, but amelanotic appearance is also possible [13]. Amelanotic melanomas pose a diagnostic dilemma and takes time to arrive at a correct diagnosis and appropriate treatment.

Norris and Taylor [14] have established diagnostic criteria for primary cervical melanomas [4] [5]:

1) Absence of melanoma elsewhere in the body,

2) Demonstration of junctional activity in the cervix,

3) Metastasis according to the pattern of cervical carcinoma.

Among the 88, only 26 cases showed junctional activity. This may be due the fact that, most often, the overlying epithelium is ulcerated. Melanin pigment is seen within the cytoplasm of neoplastic cells in most of the cases. But in 15 cases, pigment was absent in the initial small biopsy or excision making the diagnosis difficult. In such cases, the differential diagnoses included poorly differentiated carcinoma, sarcoma or lymphoma, warranting a panel of immunohistochemical stains to arrive at a definite diagnosis. Literature gives a small series of cases of amelanotic melanomas initially misdiagnosed as anaplastic carcinoma, clear cell carcinoma, chorioepithelioma, sarcoma and lymphoma [15]. In our case, the initial small biopsy was amelanotic, misleading us to a diagnosis of the most common primary entity seen here, that is, large cell non keratinizing squamous cell carcinoma. 
Table 1. Detailed review of literature about cases of primary cervical melanomas.

\begin{tabular}{|c|c|c|c|c|c|c|c|}
\hline No. & Case & $\begin{array}{c}\text { Junctional } \\
\text { activity }\end{array}$ & $\begin{array}{l}\text { Melanin } \\
\text { pigment } \\
\text { in cells }\end{array}$ & Surgery & $\begin{array}{l}\text { Chemo } \\
\text { therapy }\end{array}$ & $\begin{array}{l}\text { Radio } \\
\text { therapy }\end{array}$ & Survival \\
\hline 1 & 1889 & No & Not known & Yes & Not known & Not known & Not known \\
\hline 2 & 1923 & No & Not known & Not known & No & No & $<1$ year \\
\hline 3 & 1944 & No & Not known & Yes & No & No & $>2 \mathrm{yrs}$ \\
\hline 4 & 1950 & No & Not known & Yes & No & Yes & $<1$ year \\
\hline 5 & 1954 & Not known & Not known & Not known & Not known & Not known & Not known \\
\hline 6 & 1954 & Present & Not known & Yes & No & Yes & $<2 \mathrm{yrs}$ \\
\hline 7 & 1959 & Not known & Not known & Yes & No & Yes & $<2$ yrs \\
\hline 8 & 1959 & Present & Not known & No & No & Yes & $<1 \mathrm{yr}$ \\
\hline 9 & 1961 & Present & Not known & Yes & No & No & Not known \\
\hline 10 & 1961 & Present & Not known & No & No & Yes & $<1 \mathrm{yr}$ \\
\hline 11 & 1966 & Not known & Present & Yes & No & Yes & $<2$ yrs \\
\hline 12 & 1967 & Present & Present & No & No & Yes & Not known \\
\hline 13 & 1967 & Not known & Not known & Yes & No & Yes & $1 \mathrm{yr}$ \\
\hline 14 & 1970 & Not known & Not known & Yes & No & Yes & $>1 \mathrm{yr}$ \\
\hline 15 & 1970 & Not known & Present & Yes & No & Yes & $>1 \mathrm{yr}$ \\
\hline 16 & 1971 & Present & Present & Yes & Yes & Yes & $>1 \mathrm{yr}$ \\
\hline 17 & 1976 & No & Present & Yes & Yes & Yes & $<1 \mathrm{yr}$ \\
\hline 18 & 1979 & Not known & Not known & Yes & No & No & $1 \mathrm{yr}$ \\
\hline 19 & 1979 & Not known & Not known & Yes & Yes & Yes & $>1 \mathrm{yr}$ \\
\hline 20 & 1979 & No & Not known & Yes & Yes & No & $>1 \mathrm{yr}$ \\
\hline 21 & 1980 & Present & Present & Yes & No & No & $<1 \mathrm{yr}$ \\
\hline 22 & 1981 & Present & Not known & Yes & Yes & No & $<1 \mathrm{yr}$ \\
\hline 23 & 1981 & Present & No & Yes & No & No & Not known \\
\hline 24 & 1981 & Present & Present & Yes & No & No & $>1 \mathrm{yr}$ \\
\hline 25 & 1986 & Present & Present & Yes & No & No & $>1 \mathrm{yr}$ \\
\hline 26 & 1987 & No & No & Yes & No & Yes & $<1 \mathrm{yr}$ \\
\hline 27 & 1988 & Not known & Present & No & Yes & Yes & not known \\
\hline 28 & 1988 & Not known & Present & No & No & Yes & $<1 \mathrm{yr}$ \\
\hline 29 & 1989 & Not known & Present & Yes & No & Yes & $>1 \mathrm{yr}$ \\
\hline 30 & 1989 & Not known & Present & Yes & Yes & No & $<1 \mathrm{yr}$ \\
\hline 31 & 1990 & Present & Present & Yes & No & Yes & $>1 \mathrm{yr}$ \\
\hline 32 & 1990 & Present & Present & Yes & No & No & Not known \\
\hline 33 & 1990 & Present & Present & Yes & No & No & $>1 \mathrm{yr}$ \\
\hline 34 & 1991 & Not known & Present & Yes & No & Yes & $>1 \mathrm{yr}$ \\
\hline 35 & 1991 & Not known & Not known & Yes & Yes & Yes & $<1 \mathrm{yr}$ \\
\hline 36 & 1992 & Not known & Present & No & No & Yes & $>1 \mathrm{yr}$ \\
\hline 37 & 1992 & Not known & No & Yes & Yes & Yes & $1 \mathrm{yr}$ \\
\hline 38 & 1992 & Not known & Not known & Yes & No & No & Not known \\
\hline 39 & 1993 & Not known & Present & Yes & No & Yes & $>1 \mathrm{yr}$ \\
\hline 40 & 1995 & Not known & Present & Yes & No & No & $>1 \mathrm{yr}$ \\
\hline 41 & 1995 & Not known & Not known & Yes & No & No & $>1 \mathrm{yr}$ \\
\hline 42 & 1996 & Present & Not known & Yes & No & Yes & Not known \\
\hline
\end{tabular}




\section{Continued}

\begin{tabular}{|c|c|c|c|c|c|c|c|}
\hline 43 & 1997 & Not known & Present & Yes & Yes & No & Not known \\
\hline 44 & 1997 & No & No & Yes & Yes & No & $>1 \mathrm{yr}$ \\
\hline 45 & 1998 & Not known & No & No & No & Yes & $<1 \mathrm{yr}$ \\
\hline 46 & 1998 & Present & Present & Yes & No & No & $>1 \mathrm{yr}$ \\
\hline 47 & 1998 & Present & No & Yes & Yes & No & $>1 \mathrm{yr}$ \\
\hline 48 & 1998 & No & Not known & Yes & No & Yes & $>1 \mathrm{yr}$ \\
\hline 49 & 1999 & Not known & Present & Not known & No & Yes & $<1 \mathrm{yr}$ \\
\hline 50 & 1999 & Not known & Present & Yes & No & No & $>1 \mathrm{yr}$ \\
\hline 51 & 1999 & Not known & Present & Yes & Yes & No & $<1 \mathrm{yr}$ \\
\hline 52 & 1999 & Present & Present & Yes & No & Yes & $>1 \mathrm{yr}$ \\
\hline 53 & 1999 & Present & No & Yes & Yes & No & $<1 \mathrm{yr}$ \\
\hline 54 & 2000 & Not known & Present & No & No & Yes & $<1 \mathrm{yr}$ \\
\hline 55 & 2001 & No & No & Yes & No & No & $<1 \mathrm{yr}$ \\
\hline 56 & 2001 & Not known & Present & Yes & No & No & $<1 \mathrm{yr}$ \\
\hline 57 & 2001 & Not known & Present & Yes & No & Yes & $2 \mathrm{yrs}$ \\
\hline 58 & 2002 & Not known & Not known & Yes & No & No & $<1 \mathrm{yr}$ \\
\hline 59 & 2003 & Not known & Present & Yes & No & No & $<1 \mathrm{yr}$ \\
\hline 60 & 2003 & Not known & Present & Yes & Yes & No & $<1 \mathrm{yr}$ \\
\hline 61 & 2003 & Not known & Present & Yes & Yes & No & $<1 \mathrm{yr}$ \\
\hline 62 & 2004 & Not known & Present & Not known & Not known & Not known & Not known \\
\hline 63 & 2005 & Not known & Present & Yes & Yes & Yes & $<1 \mathrm{yr}$ \\
\hline 64 & 2005 & Not known & Not known & Not known & Not known & Not known & Not known \\
\hline 65 & 2005 & Not known & Not known & Yes & Yes & No & $>1 \mathrm{yr}$ \\
\hline 66 & 2005 & Not known & No & Yes & Yes & Yes & 2 yrs \\
\hline 67 & 2006 & Present & Present & Yes & No & Yes & 2 yrs \\
\hline 68 & 2007 & Not known & No & Yes & No & No & $>1 \mathrm{yr}$ \\
\hline 69 & 2008 & Present & Present & Yes & Yes & No & $>1 \mathrm{yr}$ \\
\hline 70 & 2009 & Present & Present & Yes & Yes & Yes & $>1 \mathrm{yr}$ \\
\hline 71 & 2009 & Not known & Present & Yes & Yes & Yes & $>1 \mathrm{yr}$ \\
\hline 72 & 2010 & Rajan duggal & Present & No & No & No & Not known \\
\hline 73 & 2011 Luz & Not known & Present & Yes & No & Yes & $>1 \mathrm{yr}$ \\
\hline 74 & 2012 El Fatemi Hinde & Not known & Not known & No & Yes & No & Not known \\
\hline 75 & 2012 Ya Ju Tsai & Not known & Not known & Yes & No & Yes & Not known \\
\hline 76 & 2013 E.Myriokef alitaki & Not known & Not known & Yes & No & No & $>1 \mathrm{yr}$ \\
\hline 77 & 2013 Nilanch ali & Present & Present & Yes & Yes & No & $<1 \mathrm{yr}$ \\
\hline 78 & 2013 Hemalat ha & Present & Present & Not known & Not known & Not known & Not known \\
\hline 79 & 2013 Not knowny anot known & Present & Present & Not known & Not known & Not known & Not known \\
\hline 80 & 2013 K J Min & Not known & Not known & Yes & Yes & Yes & $>1 \mathrm{yr}$ \\
\hline 81 & 2014 Preethi bhagel & Not known & Present & Yes & Yes & Yes & $<1 \mathrm{yr}$ \\
\hline 82 & 2014 Zhuqing & No & Present & Yes & Yes & No & $>1 \mathrm{yr}$ \\
\hline 83 & 2014 Deshpande & Not known & Present & Not known & Not known & Not known & Not known \\
\hline 84 & 2014 Yan Du & Not known & Not known & Not known & Not known & Not known & Not known \\
\hline 85 & 2014 Shrivastava & No & No & Yes & No & Yes & $<1 \mathrm{yr}$ \\
\hline 86 & 2015 Jessica L Berger & No & No & Yes & Yes & Yes & $<1 \mathrm{yr}$ \\
\hline 87 & 2015 Caglayan Geredeli & Not known & Not known & Yes & Yes & No & $<1 \mathrm{yr}$ \\
\hline 88 & 2015 Kadir Cetinkaya & Present & Present & Yes & Yes & Yes & $>1 \mathrm{yr}$ \\
\hline 89 & Present case & Present & Present & Yes & Yes & Yes & $>1 \mathrm{yr}$ \\
\hline
\end{tabular}

REF: Cases 1-71—Ref [1]; Cases 72-88-Authors name and year given in the table. 
The large tumor in the subsequent hysterectomy specimen revealed cytoplasmic melanin. The histochemical stain, Massons Fontana, demonstrate the pigment in amelanotic cases and a Melanin bleach removes the same, from pigmented cells, confirming the nature of the pigment. Immunohistochemical stains used for confirmation are S100, HMB 45 and Melan A. There has been a long debate on the staging system to be followed in primary cervical melanoma. Now it is generally accepted that staging is to be done as per the International Federation of Gynecology and Obstetrics (FIGO) staging system for cervical cancer, and not the Clarks system as the tumor generally follows the same patterns as cervical carcinoma and the FIGO system correlates better with prognosis [1]. Due to the rarity of the tumors, there is no standard treatment established for cervical melanoma. All existing literature point to surgery as the most acceptable approach, mostly radical hysterectomy with or without pelvic lymphadenectomy and/or superior vaginectomy. Although the role of negative margins in surgery is not established, there appears to be a general consensus that the primary surgery should be aimed at negative margins [16]. Melanoma being a radioresistant tumor, radiotherapy for the cervical entity has generally been reserved for positive lymphnodes, parametrial infiltration and positive margins, in addition to palliation of recurrent and residual disease. In our case, initial concurrent chemoradiation followed by extended brachytherapy did not yield the response expected of a squamous cell carcinoma, prompting surgical resction. The extended brachytherapy might have helped in achieving a satisfactory loco regional control of the disease. As with other modalities, the role of chemotherapy in these tumors is not well studied; the same chemotherapy protocols as in melanoma of the skin are being used for advanced and recurrent cases .Combined treatment modalities with surgery sandwiched between neoadjuvant chemotherapy and adjuvant chemoradiation have also been reported [17]. As per existing literature, primary melanoma of the cervix is a highly aggressive tumour which rapidly recurs and metastasizes widely, with majority deaths occurring within 3 years of diagnosis. In the cases reviewed, 65 cases underwent surgery, 26 patients received chemotherapy and 33 patients underwent radiotherapy. Caldero et al. reported a case of cervicovaginal melanoma treated with total infra levator pelvic exenteration and adjuvant radiotherapy [18]. They found a longer disease free survival of more than 8 years, and propose ultraradical surgery with adjuvant RT as the initial approach. Of the 73 patients, in whom survival data is available, 48 i.e., $65 \%$ showed a survival of one year or less and only 25 survived for more than a year (13 months to 168 months).

Primary amelanotic melanoma of the female genital tract usually has an even poorer outcome as a consequence of delayed diagnosis and lack of standardized treatment. Compared with amelanotic melanoma of the vulva, disease of the cervix is more likely to be misdiagnosed, identified at a later stage, and has a poorer outcome.

\section{Conclusion}

To conclude, we present the cases of cervical melanocytic lesions from our institute, during a period of last 6 years. The blue nevi are extremely rare incidental findings and are detected only in $0.087 \%$ of hysterectomies. Only one case of primary cervical melanoma was diagnosed, the initial small biopsy of which was labeled as poorly differentiated carcinoma. This emphasizes the rarity of this tumour and the need of ancillary techniques in accurately diagnosing poorly differentiated tumours especially in small cervical biopsies. Also malignant melanoma should be included in the list of differential diagnoses along with carcinomas and sarcomas in poorly differentiated cervical neoplasms. A detailed review of the literature shows that primary cervical melanoma is a highly aggressive tumour with poor prognosis. A consensus on treatment modalities has not yet been reached. And the overall survival is one year or less in majority of the patients.

\section{References}

[1] Pusceddu, S., Bajetta, E., Carcangiu, M.L., Formisano, B., Ducceschi, M. and Buzzoni, R. (2012) A Literature Overview of Primary Cervical Malignant Melanoma: An Exceedingly Rare Cancer. Critical Reviews in Oncology/Hematology, 81, 185-195. http://dx.doi.org/10.1016/j.critrevonc.2011.03.008

[2] WHO Classification of Tumours of Female Reproductive Organs-IARC, 2014.

[3] Mihajlovic, M., Vlajkovic, S., Jovanovic, P. and Stefanovic, V. (2012) Primary Mucosal Melanomas: A Comprehensive Review. International Journal of Clinical and Experimental Pathology, 5, 739-753.

[4] Taylor, C.E. and Tuttle, H.K. (1944) Melanocarcinoma of the Cervix Uteri or Vaginal Vault. Archives of Pathology, 38, 60-61. 
[5] Zevallos-Giampietri, E.A. and Barrionuevo, C. (2004) Common Blue Nevus of the Uterine Cervix: Case Report and Review. Applied Immunohistochemistry \& Molecular Morphology, 12, 79-82. http://dx.doi.org/10.1097/00129039-200403000-00015

[6] Uehara, T., Izumo, T., Kishi, K., Takayama, S. and Kasuga, T. (1991) Stromal Melanocytic Foci (“Blue Nevus”) in Step Sections of the Uterine Cervix. Acta Pathology Journal, 41, 751-756. http://dx.doi.org/10.1111/j.1440-1827.1991.tb03347.x

[7] Saikia, U.N., Dey, P., Saikia, B. and Gupta, I. (2004) Melanin Containing Cells of the Uterine Cervix and a Possible Histogenesis: A Case Report. Indian Journal of Pathology and Microbiology, 47, 22-23.

[8] Patel, D.S. and Bhagavan, B.S. (1985) Blue Nevus of the Uterine Cervix. Human Pathology, 16, 79-86. http://dx.doi.org/10.1016/S0046-8177(85)80217-3

[9] Sahin, S., Seckin, S. and Seckin, L. (2015) Blue Nevus of the Cervix, an Unusual Lesion-A Case Report. International Journal of Pathology, 13, 36-40.

[10] Dahlgren, L., Schedvins, K., Kanter-Lewensohn, L., Dalianis, T. and Ragnarsson-Olding, B.K. (2005) Human Papilloma Virus (HPV) Is Rarely Detected in Malignant Melanomas of Sun Sheltered Mucosal Membranes. Acta Oncologica, 44, 694-699. http://dx.doi.org/10.1080/02841860500247461

[11] Holmstrom, M. and Lund, V.J. (1991) Malignant Melanomas of the Nasal Cavity after Occupational Exposure to Formaldehyde. British Journal of Industrial Medicine, 48, 9-11. http://dx.doi.org/10.1136/oem.48.1.9

[12] Mc Laughlin, C.C., Wu, X.C., Jemal, A., et al. (2005) Incidence of Noncutaneous Melanomas in the US. Cancer, 103, 1000-1007. http://dx.doi.org/10.1002/cncr.20866

[13] Cantuaria, G., Angioli, R., Nahmias, J., Estape, R. and Penalver, M. (1999) Primary Malignant Melanoma of the Uterine Cervix: Case Report and Review of the Literature. Gynecologic Oncology, 75, 170-174. http://dx.doi.org/10.1006/gyno.1999.5491

[14] Norris, H.J. and Taylor, H.B. (1966) Melanomas of the Vagina. American Journal of Clinical Pathology, 46, 420-426. http://dx.doi.org/10.1093/ajcp/46.4.420

[15] Duggal, R. and Srinivasan, R. (2010) Primary Amelanotic Melanoma of the Cervix: Case Report with Review of Literature. Gynecologic Oncology, 21, 199-202. http://dx.doi.org/10.3802/jgo.2010.21.3.199

[16] Piura, B. (2008) Management of Primary Melanoma of the Female Urogenital Tract. The Lancet Oncology, 9, $973-981$. http://dx.doi.org/10.1016/S1470-2045(08)70254-7

[17] Min, K.-J., Kim, Y.-S., Hong, J.-H., Lee, J.-K. and Yang, D.-S. (2014) Primary Malignant Melanoma of Uterine Cervix: A Suggestion of New Scheme of Treatment Combination. Chinese Journal of Cancer Research, 26, 351-354.

[18] Salazar, L.C., de Leon, D.C., Montiel, D.P., Almogabar-Villagrá, E., Villavicencio, V. and Cetina, L. (2011) Primary Malignant Melanoma of the Uterine Cervix Treated with Ultraradical Surgery: A Case Report. International Scholarly Research Network ISRN Obstetrics and Gynecology. 